\title{
The physics of life
}

The flow of ideas from physics to elsewhere has a long and sometimes chequered history. Niels Bohr famously speculated that the uncertainty principle of quantum physics might be useful in psychology, where observation unavoidably influences an individual's behaviour. Some artists drew on the concepts of Einstein's theory of relativity and applied them - with terrific distortion, obviously - to explain the relativity of moral values. Einstein thought there was something 'psychopathological' about such efforts. "The present fashion of applying the axioms of physical science to human life", he said, "is not only a mistake but has something reprehensible to it."

Fortunately, things are rather different today. We've lost the naive belief that physical laws might have direct analogues in the human world, yet have gained confidence that the methods of physics, applied cautiously, can really help. Physicsbased modelling of financial markets, to take one example, grows more impressive day by day.

In the early 1990s, economist Brian Arthur introduced a model to explore situations in which people have to make decisions, yet cannot do so on the basis of calculation. He imagined a popular restaurant with live music every Thursday evening. Although many people would like to go, if too many go, the place is overcrowded and everyone is miserable. Hence the problem: it's good to go when most other people don't go, and to stay home when they do go. There's no rational way to decide, as the wisdom of a choice depends ultimately on what other people do.

Drawing on earlier psychological studies, Arthur supposed that real people handle such problems in an adaptive way. We keep a handful of 'strategies' in our heads about how past bar attendance might predict future attendance, and make decisions by using whichever has been most accurate in the recent past. Arthur programmed a computer to simulate a group of people facing this problem, and found that as individuals adapted, attendance ended up fluctuating about the crowding threshold.

A number of physicists have since used this model to explore more realistic social systems, including financial markets. A financial market is clearly not a restaurant, yet there are similarities. The wisdom of a decision now,

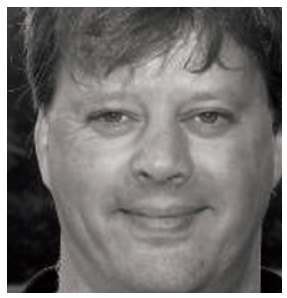

The methods of physics, applied cautiously, can really help. to buy or sell stock, depends not only on the real facts about the company in question, but on the unpredictable decisions of many others. Also, many investors look to the record of past price movements to predict what will happen next.

Work in this area has led to a variety of increasingly sophisticated models, that give realistic results when compared with empirical reality. They reproduce the statistical character of real market fluctuations, for example, and have even been used to predict market movements with some success (for a recent review, see http://arxiv.org/ abs/physics/0606107). Intriguingly, researchers have found surprising links to physics. These models show phase transitions in the collective market organization, depending on the number of participating people, and such transitions can be described analytically using the replica trick of spin-glass theory.

This excursion from physics into the social sciences shows real promise - not just hope and hype - for building a fundamental understanding of collective human systems. I don't think Einstein would find anything 'psychopathological' in that.

Mark Buchanan

\section{The end of the world as we know it}

It took the Earth and the Sun hundreds of millions of years to produce the world's petroleum in 150 years we've used up nearly half of it. With worldwide oil consumption rapidly expanding, we are already burning up 25 billion barrels a year. We will soon start to run out of oil.

With any natural resource, the amount available starts at zero when it's first discovered, rises to a maximum that will never be exceeded, and then declines forever. As the demand for oil only goes on increasing, we will be in trouble when we reach the maximum, not when we pump the last barrel of oil out of the ground. That should occur (by symmetry) roughly when we reach the halfway point in our use of the stuff. We are already very close to the halfway point.

Of course, all fossil fuels are replaceable. Natural gas could replace oil, but the worldwide peak for natural gas is only a decade or two behind the oil peak, so that's a temporary solution at best. Other possible substitutes for oil include shale oil, methane hydrate and coal: all of these have very serious drawbacks. Another possibility is biofuels, such as ethanol or biodiesel, but it's far from obvious that their use could be scaled up sufficiently to replace oil. And whatever does replace oil will probably continue to pour greenhouse gases into the atmosphere. We are doing an

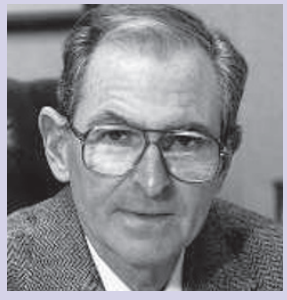

We are doing an uncontrolled experiment with the climate of the only planet we have. uncontrolled experiment with the climate of the only planet we have. It is very foolish indeed.

What is desperately needed is a crash programme to enable us to kick the fossil-fuel habit now, before we use all the stuff up. The fact is, we are very good at solving technical problems once we put our minds and resources on the line. But we need courageous, visionary political leadership that will challenge us scientists to do it - just as JFK once challenged us to put a man on the Moon. Unfortunately, that seems unlikely. But the alternative is nothing less than the end of our oil-based civilization. 